Ann. Biol. anim. Bioch. Biophys., I974, 14 (2), 363-369.

\title{
SÉPARATION DES SPERMATOZOÏDES MOBILES DES AUTRES CELLULES ET DES DÉBRIS CELLULAIRES PRÉSENTS DANS LE SPERME BOVIN
}

\author{
C. PRAKASH (1) \\ Laboratoire de Génétique biochimique, \\ Centre national de Recherches zootechniques, I. N. R. A., \\ 78350 Jouy en Josas \\ RÉSUMÉ
}

Nous décrivons dans ce travail une technique de centrifugation du sperme de taureau en gradient de ficoll qui permet d'obtenir des suspensions de spermatozoïdes dépourvues de cellules ou débris cellulaires diploïdes, et soulignons l'intérêt d'utiliser de telles préparations dans l'étude immunologique des spermatozoïdes.

\section{INTRODUCTION}

Un des facteurs qui font obstacle aux recherches immunologiques sur les spermatozoïdes est la présence, dans le sperme, d'autres cellules et de débris cellulaires. Dans plusieurs expériences décrites dans les publications qui traitent de l'absorption, de l'immunisation et de la fixation du complément par des spermatozoïdes de diverses espèces de mammifères, les résultats démontrant l'existence de certains antigènes sur les spermatozoïdes, cellules haploïdes, prêtent à critique à cause de la présence de cellules diploïdes et de débris cellulaires dans les préparations. De même, les résultats des réactions d'agglutination mixte ou d'agglutination d'anti-globulines mixtes, qui devraient être positifs, apparaissent comme négatifs en raison de l'affinité plus grande des cellules indicatrices pour les cellules autres que les spermatozoïdes et pour les débris cellulaires. D'autre part, dans les tests de cytotoxicité sur des spermatozoïdes, l'interprétation des résultats est rendue difficile par un " bruit de fond " relativement élevé dû à la présence de spermatozoïdes morts. Ennfin, les effets de la "stimulation mixte de lymphocytes et de spermatozoïdes " sont également difficiles à interpréter. India.

(1) Adresse actuelle : Division of Genetics, National Dairy Research Institute, Karnal, Haryana, 
Notre expérience nous a montré que ni le lavage à l'aide de solutions isotoniques contenant de l'albumine, ni la sédimentation en solution tamponnée n'étaient des techniques satisfaisantes (préparation insuffisamment propre, surtout dans le premier cas, et motilité réduite par le traitement).

Nous décrivons, dans le présent travail, une méthode simple et rapide pour séparer, en gradient de ficoll, les spermatozoïdes mobiles des spermatozoïdes immobiles ou morts, ainsi que des cellules et débris cellulaires contenus dans le sperme de taureau.

\title{
MATÉRIEL
}

\author{
A. - Produits
}

L'origine des produits est la suivante : Ficoll, Pharmacia (PM estimé par diffusion de la lumière 400 ooo ; chlorures, évalués en $\mathrm{NaCl},<$ I p. Ioo) ou Sigma (PM indiqué 400 ooo enr., $\mathrm{NaCl}$ o,3 p. Ioo environ), ce dernier n'ayant été utilisé que dans les expériences initiales; diméthyldichlorosilicane, Biorad; albumine sérique bovine (fraction $\mathrm{V}$ de $\mathrm{Cohn}$ ), ${ }^{14} \mathrm{C}$ D-glucose, C. E. A., Saclay (activité spécifique de $170 \mathrm{mci} / \mathrm{mM}$ et pureté radiochimique de 98 p. roo; réf. $\mathrm{CBl}(\mathrm{F})$, lot $\left.\mathrm{n}^{0} 4965\right)$; ce glucose ${ }^{14} \mathrm{C}$ est utilisé après dissolution dans le milieu I99, à raison de I,I mg soit I mci par ml. Les solutions tampons ont été préparées avec de l'eau récemment redistillée dans du verre et conservée à l'état congelé. Toutes les manipulations du sperme ont été effectuées dans de la verrerie siliconée ou des récipients et instruments en matière plastique.

Pour toutes les dilutions et pour le lavage du sperme, nous avons utilisé le tampon Tris, modifié selon Bhargava (I97I) : Tris o,oI6 $\mathrm{M} ; \mathrm{NaCl}$ I I $7 \mathrm{mM} ; \mathrm{KCl}_{5} \mathrm{mM} ; \mathrm{MgSO}_{4}^{\prime} \mathrm{I}, \mathrm{I} \mathrm{mM} ; \mathrm{NaH}_{2}$ $\mathrm{PO}_{4} \mathrm{I}, \mathrm{o} \mathrm{mM} ; \mathrm{NaHCO}_{3} 26 \mathrm{mM}$; glucose o, I p. ıoo, pénicilline ıоo UI/ml; BSA $2,5 \mathrm{~g} / \mathrm{l} ; \mathrm{pH}$ 7,5). Pour débarrasser le ficoll du sel qu'il renferme, nous en faisons une solution concentrée (30 à 40 p. Ioo) dans de l'eau distillée, et dialysons cette dernière à $4^{\circ} \mathrm{C}$ contre quarante volumes d'eau distillée contenant ıoo UI de pénicilline par millilitre ; après trois renouvellements de l'eau distillée, la solution de ficoll dialysée est lyophilisée ; le lyophilisat pulvérisé au mortier est conservé dans un flacon à fermeture hermétique. La concentration osmotique de $\mathrm{I}_{4} \mathrm{~g}$ par $\mathrm{Ioo} \mathrm{ml}$ de tampon de la solution de ficoll et du tampon Tris est ajustée à 285 milliosmoles par litre à l'aide de l'osmomètre Fiske. La solution de ficoll, répartie en fractions de $2 \mathrm{ml}$ dans des tubes de matière plastique gradués (diamètre I I mm, longueur $75 \mathrm{~mm}$, volume $6 \mathrm{ml}$ ), est conservée à l'état congelé jusqu'à usage.

\section{B. - Collecte et traitement du sperme}

Le sperme de taureau est collecté au moyen d'un vagin artificiel, et placé dans une bouteille isolante. Le sperme de 3 taureaux entretenus au C. N. R. Z. à Jouy en Josas, et de 30 taureaux des Centres d'insémination artificielle de l'Aigle et de Mayenne a été utilisé.

\section{DESCRIPTION DE LA MÉTHODE ET DISCUSSION}

Ie sperme est refroidi progressivement jusqu'à $20^{\circ} \mathrm{C}$, au bain-marie, en 30 à 90 minutes. Simultanément, la solution de ficoll et le tampon congelé sont réchauffés, ret amenés à la même température. On ajoute alors lentement et en agitant doucement un volume du sperme à 3 volumes de tampon. Quatre millilitres de sperme ainsi dilué sont ensuite déposés avec précaution à la surface de $2 \mathrm{ml}$ de solution de ficoll, contenus dans un tube maintenu incliné pendant cette opération. Ce dernier est alors 
soumis à une centrifugation à $600 \mathrm{~g}$ pendant $\mathrm{I} 5$ minutes à la température ambiante (fig. I) ; après cette centrifugation, on aspire soigneusement le surnageant, la couche intermédiaire et $0,5 \mathrm{ml}$ de la couche immédiatement sous-jacente, et l'on transfère le reliquat $(\mathrm{r}, 5 \mathrm{ml})$ dans un tube propre, pour éviter toute contamination par des résidus de la couche supérieure. On veille également à laisser quelques gouttes au fond du tube pour éviter une contamination par les spermatozoïdes morts qui s'y trouvent toujours, comme nous le verrons plus loin. L'examen de gouttes prélevées dans les diverses zones du tube après centrifugation a été effectué soit entre lame et lamelle, soit sur frottis sec, à l'aide d'un microscope à contraste de phase : la couche inférieure contient plus de $9 \circ \mathrm{p}$. roo de spermatozoïdes immobiles ou morts ; au-dessus $(0,3 \mathrm{~cm})$ se trouve une couche très riche en spermatozoïdes mobiles; dans la couche intermédiaire formée de liquide séminal dilué et de ficoll, on observe des débris cellulaires et des cellules autres que les spermatozoïdes ; la couche suivante contient surtout des petites particules; enfin la zone supérieure contient quelques cellules, de petites particules et de la vaseline qui provient du vagin artificiel. Sur les frottis colorés au Giemsa, quelques cellules de la couche intermédiaire présentaient la morphologie de lymphocytes.

Des essais entrepris en faisant varier la concentration de ficoll ( 5 à $25 \mathrm{~g}$ pour Ioo $\mathrm{ml}$ ), la vitesse (300 à $3000 \mathrm{~g}$ ) et la durée de centrifugation $(5$ à $60 \mathrm{mn}$ ) ont montré qu'on obtient la meilleure séparation en utilisant $14 \mathrm{~g}$ de ficoll Pharmacia pour $100 \mathrm{ml}$ de tampon Tris (densité I,05 g/ml ; viscosité par rapport à l'eau Io) et une centrifugation à $600 \mathrm{~g}$ pendant $\mathbf{r} \mathrm{mn}$ (fig. I). La concentration optimale en ficoll dépend du lot utilisé.

De manière générale, à moins que le sperme ne soit abondamment chargé en débris cellulaires ou en cellules autres que les lymphocytes, on peut, par cette méthode, obtenir, dans la couche de ficoll, des spermatozoïdes "propres ", avec une perte de Io à $20 \mathrm{p}$. Ioo dans l'interphase ou dans le surnageant. Les spermatozoïdes se meuvent alors dans la solution de ficoll comme des cellules isolées, libres, pourvu que la mobilité initiale ait été bonne. Il arrive cependant que 1'on observe 1'agglutination dans le ficoll de quelques spermatozoïdes. Selon une revue de CoHEN (I97I) le phénomène d'agglutination des spermatozoïdes de taureaux dans l'éjaculat et dans le diluant est occasionnel puisque les spermatozoïdes d'un même sujet peuvent, selon l'éjaculat, s'agglutiner ou ne pas s'agglutiner. Nous considérons que l'agglutination que nous avons observée dans le ficoll relève du même phénomène et n'est donc pas imputable à la technique que nous préconisons. $\mathrm{L}_{\text {a }}$ floculation des spermatozoïdes de lapins dans le ficoll observée par BRANHAM (r970) dans ses essais de fractionnement de diverses populations de spermatozoïdes peut être attribuée à la forte concentration de ficoll utilisée dans ce travail.

L'addition de nigrosine que recommande cet auteur, beaucoup plus élevéz que celle que nous utilisons, nous semble devoir être évitée, afin de limiter les risques d'altération des préparations.

L'efficacité d'une méthode de séparation des spermatozoỉdes se mesure non seulement au degré d'élimination des débris cellulaires et des spermatozoïdes morts, mais aussi au degré d'élimination du plasma séminal. Pour estimer la quantité de plasma séminal qui pénètre dans le gradient de ficoll, nous avons mis en œuvre deux techniques différentes: 


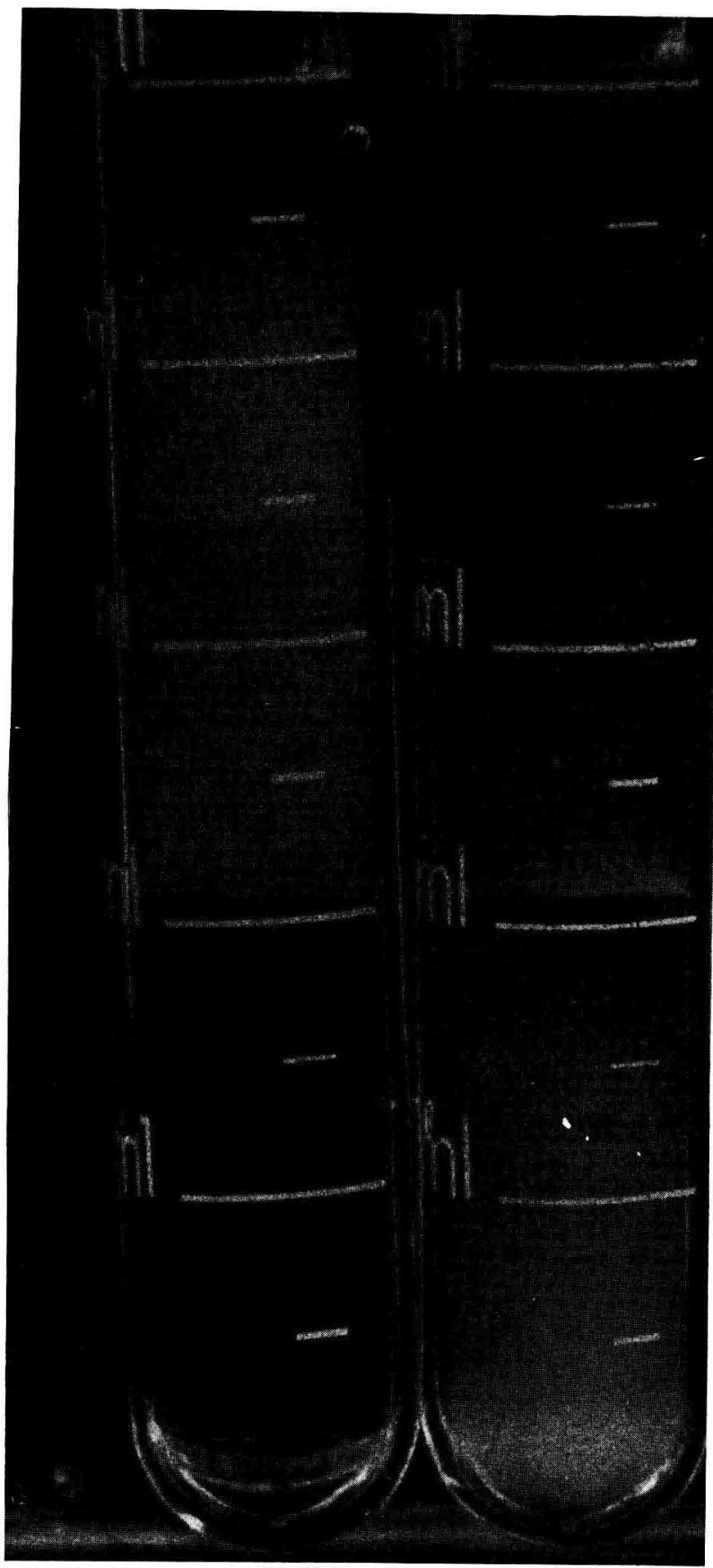

FIG. I. - Séparation des spernatozoïdes sur couche de ficoll.

A gauche: tube avant centrifugation $(3 \mathrm{ml}$ de sperme dilué déposés sur $2 \mathrm{ml}$ de ficoll) A droite : tube après centrifugation. 


\section{A. - Mesure de radioactivité après adjonction de glucose ${ }^{14} \mathrm{C}$}

Avant d'être soumis à la technique de fractionnement dans un gradient de ficoll que nous venons de décrire, le sperme est additionné d'une solution de glucose ${ }^{14} \mathrm{C}$ dans du tampon Tris. Le liquide séminal dilué et la couche de ficoll sont recentrifugés à $3000 \mathrm{~g}$ pendant 30 minutes, et à $4^{\circ} \mathrm{C}$, après quoi des parties aliquotes de chacun d'eux sont soumises au comptage de radioactivité dans un spectromètre à scintillation Packard Tri-Carb. Pour ces essais, nous avons utilisé deux dilutions différentes :

I-4 $\mathrm{ml}$ de sperme ajoutés à $\mathrm{I} 2 \mathrm{ml}$ de tampon Tris contenant roo $\mu 1$ d'une solution de glucose ${ }^{14} \mathrm{C}$; cette dernière est elle-même obtenue en diluant Ioo fois dans le tampon Tris roo $\mu 1$ de la solution mère de glucose ${ }^{14} \mathrm{C}$ dans le milieu 199.

2-6 $\mathrm{ml}$ de sperme ajoutés à $\mathrm{I} 8 \mathrm{ml}$ de tampon Tris contenant Ioo $\mu 1$ de solution mère de glucose ${ }^{14} \mathrm{C}$ dans le milieu 199.

Les résultats de ces essais, qui figurent dans le tableau I, indiquent que la couche de ficoll, dans laquelle se trouvent les spermatozoïdes mobiles, contient moins de 2 p. roo de la radio-activité totale.

\section{TABLEAU I}

Estimation du degré de contamination par le plasma séminal, de la couche de ficoll contenant les spermatozoïdes mobiles

(Méthode au glucose ${ }^{14} \mathrm{C}$ )

\begin{tabular}{|c|c|c|c|}
\hline \multirow{2}{*}{$\begin{array}{c}\text { No de } \\
\text { l'expérience }\end{array}$} & \multicolumn{2}{|c|}{$\begin{array}{l}\text { Radioactivité en coups } \\
\text { par minute et par millilitre } \\
\text { (Moyenne de deux répétitions) }\end{array}$} & \multirow{2}{*}{$\begin{array}{c}\text { Rapport : } \\
\frac{\text { couche de ficoll }}{\text { sperme }} \\
(\%)\end{array}$} \\
\hline & $\begin{array}{c}\text { dans le sperme } \\
\text { au départ } \\
\text { (dilué 1/4) }\end{array}$ & $\begin{array}{l}\text { dans la couche } \\
\text { de ficoll après } \\
\text { fractionnement }\end{array}$ & \\
\hline 1 & 16522 & 252 & 1,53 \\
\hline 2 & 991967 & 17699 & 1,78 \\
\hline
\end{tabular}

Il a été difficile d'obtenir un surnageant net pour le comptage des scintillations. I'utilisation d'un acide aminé radioactif ou d'une autre substance soluble difficilement métabolisable donnerait sans doute une image plus claire.

\section{B. - Dosage de l'acide citrique.}

On sait que le plasma séminal contient de l'acide citrique qui provient exclusivement, tout au moins chez le taureau, de la vésicule séminale (MANN, I964). Un dosage de l'acide citrique dans la couche de ficoll après centrifugation doit donc permettre d'estimer le degré de contamination de cette couche par le plasma séminal. Nous avons effectué ce dosage en recourant à la méthode de SAFFran et DENSTEDT (1948). Les mesures d'absorption, effectuées à l'aide d'un spectrophotomètre Zeiss, 
ont été faites à $400 \mathrm{~nm}$, comme l'ont suggéré MulTamaki et NiEMI (I972). Pour ces essais, nous avons utilisé le sperme de II taureaux de race Normande, âgés de un an et demi à deux ans. Après centrifugation selon la technique dont la description fait l'objet de cette publication, les trois quarts du ficoll ( $I, 5$ sur $2 \mathrm{ml}$ ) sont aspirés par la base de la couche et transvasés dans un autre tube ; ce ficoll est alors soigneusement mélangé avant de subir le prélèvement d'une partie aliquote destinée à la déprotéinisation trichloracétique.

TABLEAU 2

Estimation du degré de contamination par le plasma séminal, de la couche de ficoll contenant les spermatozoides mobiles

(Méthode par dosage de l'acide citrique)

\begin{tabular}{c|c}
\hline $\begin{array}{c}\text { Teneur moyenne en acide citrique } \\
\text { par millilitre } \\
\text { (moyenne de 11 échantillons) }\end{array}$ & $\begin{array}{c}\text { Rapport : } \\
\text { couche de ficoll }\end{array}$ \\
\begin{tabular}{c|c} 
dans le sperme \\
au départ (dilué 1/4)
\end{tabular} & $\begin{array}{c}\text { dans la couche de ficoll } \\
\text { après fractionnement } \\
(\%)\end{array}$ \\
\hline $1812,0 \mu g \pm 21,0$ & $52,5 \mu \mathrm{g} \pm 15,0$
\end{tabular}

Les résultats de nos mesures qui figurent dans le tableau 2, indiquent que la couche de ficoll ne contient que I,3 p. Ioo de la quantité totale d'acide citrique présente dans la semence entière. Rappelons ici que les spermatozoïdes eux-mêmes adsorbent une petite quantité d'acide citrique ; après trois lavages des spermatozoïdes selon une technique qui peut être considérée sur ce point comme équivalente à la nôtre, Multamaki et Niemi (I972), ont estimé que la quantité d'acide citrique restant adsorbé représentait $0,06 \mathrm{p}$. Ioo de la quantité présente dans la semence entière. L'ensemble de ces données confirme donc l'efficacité de notre méthode. Si l'on souhaite réduire encore la contamination des spermatozoïdes par le plasma séminal, on peut répéter le fractionnement sur gradient de ficoll à partir de la couche de ficoll de la première opération. Il est préférable, dans ce cas, de diluer I ml provenant de la partie inférieure de la couche de ficoll avec $3 \mathrm{ml}$ de tampon Tris et de déposer ce mélange sur une couche fraîche de $2 \mathrm{ml}$ de ficoll.

Nous avons recherché si l'on pouvait observer des figures méïotiques au niveau des cellules que notre technique sépare dans la couche intermédiaire et dans le surnageant. Ces cellules ont été préparées selon le protocole suivant : centrifugation de la couche intermédiaire et du surnageant à I $500 \mathrm{~g}$ pendant $30 \mathrm{mn}$; dispersion puis incubation du culot pendant Io $\mathrm{mn}$ à $37^{\circ} \mathrm{C}$ dans $20 \mathrm{ml}$ d'une solution aqueuse de citrate de sodium à $0,9 \mathrm{p}$. IOO ; centrifugation à I $500 \mathrm{~g}$ pendant $30 \mathrm{mn}$, mise en suspension du culot dans $20 \mathrm{ml}$ d'un mélange d'alcool éthylique et d'acide acétique $(3: \mathrm{I})$; après une nuit à $4^{\circ} \mathrm{C}$, recentrifugation à $\mathrm{I} 500 \mathrm{~g}$ pendant $30 \mathrm{mn}$. Après remise en suspension de ce culot, de petites gouttes ont été déposées sur une lame chauffée à $45^{\circ} \mathrm{C}$ environ, séchées et colorées au bleu d'Unua et de Giemsa. L,es résultats de ces essais ont été négatifs. 
SPERL,ING et KADEN (I97I) ont observé des figures méiotiques dans les cellules de liquide séminal de sujets tant normaux qu'oligospermiques. Le fait que nous n'ayons pu observer de telles figures nous semble devoir être attribué aux techniques de traitement et de coloration des cellules; il semble exclu, en effet, que certaines d'entre elles aient pu être éliminées lors des opérations de fractionnement.

Reçu pour publication en juillet 1973.

\section{REMERCIEMENTS}

Nous remercions M. F. Grosclaude qui nous a accueilli dans son Laboratoire et constamment aidé au cours de ce travail.

Nous remercions MM. R. L. Cerriani, C. Delouis et R. H. F. Hunter, ainsi que MM. Cassou et Lamétrie, Directeurs des Centres d'Insémination de l'Aigle et de Mayenne pour l'aide qu'ils nous ont apportée.

\section{SUMMARY}

\section{SEPARATION OF MOTILE SPERMATOZOA FROM OTHER CELIS AND CELLULAR DEBRIS FOUND IN BOVINE SEMEN}

A density gradient method employing ficoll is described to prepare spermatozoa free from other cells and cellular debris found in bull semen. The necessity of using spermatozoa free from heterologous cells and cellular debris of diploid origin in immunological experiments is briefiy discussed.

\section{RÉFÉRENCES BIBLIOGRAPHIQUES}

Bhargava, I97r. (Communication personnelle.)

Branham J. M., 1970. Separation of rabbit semen into two populations of spermatozoa by centrifugation. J. Reprod. Fert., 22, 469-482.

Сонек J., r97r. The comparative physiology of gamete populations in Advances in Comparative Physiology and Biochemistry. Lowenstein O., ed., Academic Press, New York, 4, p. 287.

Dumont J. E., van Sande J., I965. Action de l'hormone thyréotrope. Bull. Chim. Biol., 47, 32 I-34I. MANN T., I964. Biochemistry of semen and of the male reproductive tract. Methuen, London, p. $29 \mathrm{I}$.

Multamaki S., Niemi M., I972. Trypsin-like proteolytic activity in an acrosomal extract of bull spermatozoa. Int. J. Fert., 17, 43-52.

Saffran M., Denstedt O., I948. A rapid method for the determination of citric acid. J. Biol. Chem., 175, $849-855$.

SPERLING K., KADEN R., I97r. Meiotic studies of the ejaculated fluid of humans with normal sperm count and oligospermia. Nature, 232, $48 \mathrm{I}$. 